Letter to Editor

\title{
Nasolabial cysts: sublabial or neumann incision?
}

\author{
Volume 3 Issue I - 2015
}

\author{
Mayte Pinilla Urraca \\ Department of Otorhinolaryngology,Autonomous University of \\ Madrid, Spain
}

Correspondence: Mayte Pinilla Urraca, Department of Otorhinolaryngology Head \& Neck Surgery, C/ Joaquin Rodrigo, 228002 Majadahonda, Madrid, Spain, Tel +34 6298I 0134 , Email pinillamayte@gmail.com

Received: June 17,2015 | Published: August 10, 2015

\begin{abstract}
Nasolabial cysts are an uncommon non-odontogenic lesions and were described in 1882 by Zuckerkandl and are also known as naso alveolar cysts. ${ }^{1}$ They probably arise from embryologic remnants of the naso lacrimal duct. ${ }^{2}$ This lesion is mostly unilateral and occurs more frequently in adult females. Their growth is slow over several years and remain undetected unless and until they become infected or are associated with facial deformity. Clinically, these cysts present as smooth, fluctuant soft-tissue masses between the upper lip and nasal aperture, with obliteration of the naso labial fold and elevation of the nasal ala ${ }^{3}$. Imaging tests, like tomography $(\mathrm{CT})$ of paranasal sinuses and magnetic resonance imaging (MRI) may be useful, showing a homogeneous cystic lesion located anterior to the piriform aperture. ${ }^{1,4}$
\end{abstract}

Despite the fact that they are soft tissue cysts and are situated extraosseously they may sometimes cause bone destruction. ${ }^{4}$ Differential diagnosis includes cysts of the nasopalatine duct, periapical inflammatory lesions (granuloma, cyst or abscess) and epidermoid or epidermal inclusion cysts. ${ }^{4}$ The aims of treatment are to prevent infection, to establish a histopathological diagnosis, and to ameliorate a cosmetic deformity. Simple aspiration, injections with sclerosing agent, destruction by cautery, endoscopic marsupialization, needle aspiration and incision and drainage, have been described in the management of these cysts, ${ }^{3}$ but complete surgical excision of the nasolabial cyst is the best treatment. ${ }^{4}$ The classical incision is sublabial. Usually the upper gingivolabial sulcus was incised just below the piriform apertures, and dissection exposed a smooth, wellcircumscribed cyst swelling superficial to the lateral half of the bony floor of the nose. ${ }^{1,3}$ In general, the cysts are easily dissected off, but dissection should be made carefully in order to avoid perforation and sensory damages. In this surgical approach, the incision coincides with the resection area, so that healing can lead to fistula and / or retraction pre maxilar.

An alternative to this incision is the Neumann incision, use by endodontists for performing alveolo plasties. ${ }^{4}$ It consists of incision in the free edge of the gingival in the region of the inter dental papillae, from the medial portion of the lateral incisor to the lateral portion of the second premolar and first molar, with two vertical extensions, one medial and other lateral to the gingival and labia with subsequent elevation of the flap created, allowing full access to the pyriform aperture (Figure 1).

After the intervention, the mucosal flap is put back into its original position and the papillae are sutured with absorbable sutures and a traumatic needle (Figure 1), as well as the vertical extensions ${ }^{4}$. This incision considers the vessels and nerves in the region therefore the local sensory disturbance and bleeding is minimal, and avoid that the incision coincides with the resection area as the sublabial incision with the probable complication described previously. Although the sublabial incision is the most used by ENT specialist, we must know Neumann incision which allows a good approach to the perform aperture and the complete naso labial cyst excision without important potential complications.

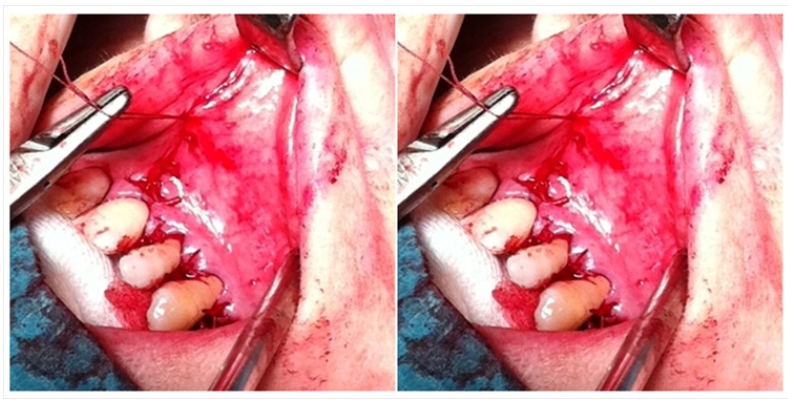

Figure I Neumann Incision.

a. Exposition of the cyst.

b. Suture of incision.

\section{Acknowledgments}

None.

\section{Conflicts of interest}

Author declares there are no conflicts of interest.

\section{Funding}

None.

\section{References}

1. Ahmet U, Hunturk A, Mesut Tezer, et al.Bilateral Nasoalveolar cyst: case report and therapeutic approach. KBB-Forum. 2007;6(2):66-68.

2. Thais Cristina VR, Ricardo AM, Ricardo SG, et al.Transnasal Approach to Marsupialization of the Naso labial Cyst: Report of 2 Cases. JOral Maxillofac Surg. 2007;65(6):1241-1243.

3. Heng WY, Cheow-YLJ, Chow-Lin YS. Nasolabial cysts: Clinical features, diagnosis, and treatment. British Journal of Oral and Maxillo facial Surgery. 2007;45(4):293-297.

4. Alexandre BO, Larissa Neri, Ingrid Helena, et al. Giant Nasolabial Cyst Treated Using Neumann Incision: Case Report. Int Arch Otorhinolaryngol. 2013;17(4):421-423. 